\title{
Enhanced anti-tumor efficacy of 5-aminolevulinic acid- gold nanoparticles-mediated photodynamic therapy in cutaneous squamous cell carcinoma cells
}

\author{
Yu-fei Chi(1) ${ }^{1,2 *}$, Jing-jing Qin (ii) ${ }^{3 *}$, Zhi Li(i) ${ }^{4}$, Qin Ge (ii) ${ }^{5}$, and Wei-hui Zeng (i) ${ }^{1}$ \\ ${ }^{1}$ Department of Dermatology, The Second Affiliated Hospital of Xi'an Jiaotong University, Xi'an, China \\ ${ }^{2}$ Department of Dermatology, Xijing Hospital, Fourth Military Medical University, Xi'an, China \\ ${ }^{3}$ Department of Dermatology, Forest Industry Worker Hospital of Shaanxi Province, Xi'an, China \\ ${ }^{4}$ Department of Dermatology, Qingdao Municipal Hospital, Qingdao, China \\ ${ }^{5}$ Department of Dermatology, Jingmen No.1 People's Hospital, Jingmen, China
}

\begin{abstract}
The objective of this study was to investigate whether the conjugation of gold nanoparticles (GNPs) to 5-aminolevulinic acid (5ALA) could enhance the anti-tumor efficiency of photodynamic therapy (PDT) in epidermoid carcinoma cells. The mRNA and protein expression levels were determined by quantitative real-time PCR and western blot, respectively. Cell viability, apoptosis, invasion, and migration were determined by MTT assay, flow cytometry, transwell invasion assay, and migration assay, respectively. Singlet oxygen generation was detected by the singlet oxygen sensor green reagent assay. Our results showed that PDT with 5-ALA and GNPs-conjugated 5-ALA (5-ALA-GNPs) significantly suppressed cell viability, increased cell apoptosis and singlet oxygen generation in both HaCat and A431 cells, and PDT with 5-ALA and 5-ALA-GNPs had more profound effects in A431 cells than that in HaCat cells. More importantly, 5-ALA-GNPs treatment potentiated the effects of PDT on cell viability, cell apoptosis, and singlet oxygen generation in A431 cells compared to 5-ALA treatment. Further in vitro assays showed that PDT with 5-ALA-GNPs significantly decreased expression of STAT3 and Bcl-2 and increased expression of Bax in A431 cells compared with PDT with 5-ALA. In addition, 5-ALA-GNPs treatment enhanced the inhibitory effects of PDT on cell invasion and migration and $W n t / \beta$-catenin signaling activities in A431 cells compared to 5-ALA treatment. In conclusion, our results suggested that GNPs conjugated to 5-ALA significantly enhanced the anti-tumor efficacy of PDT in A431 cells, which may represent a better strategy to improve the outcomes of patients with cutaneous squamous cell carcinoma.
\end{abstract}

Key words: Photodynamic therapy; 5-ALA-GNPs; Cell viability; Cell apoptosis; Cell invasion and migration; Cutaneous squamous cell carcinoma

\section{Introduction}

Cutaneous squamous cell carcinoma (cSCC) is the second most frequently diagnosed non-melanoma skin cancer worldwide (after basal cell carcinoma) (1) and the incidence of cSCC is increasing. Surgical excision remains the gold standard treatment for localized cSCC, which can be managed through excisional surgery, cryosurgery, curettage and desiccation, Mohs micrographic surgery, or micrographic surgery (2). However, these approaches have a number of side effects, such as scarring, development of sensitive areas and painful lesions, and are not suitable for elderly patients (3). Moreover, treatment of extensive locally destructive or metastatic disease is still a challenge and treatments are seldom curative. As the primary non-surgical option, radiation therapy also plays an important role in the treatment of cSCC, especially tumors on the lip and eyelid (4). However, radiation therapy commonly induces side effects including nausea, vomiting, erythema, epidermal atrophy, telangiectasia, soft-tissue necrosis, and radiation-induced malignancies that some patients find difficult to tolerate (5).

Photodynamic therapy (PDT) is an effective, noninvasive procedure that has been clinically approved for treating cancer and other malignant diseases. PDT offers the advantages of minimal invasiveness, better cosmetic outcomes, low morbidity, minimal functional disturbances, and is well tolerated and can be applied repeatedly at the same site (6). Thus, PDT is widely used in the treatment of superficial skin cancers including actinic

Correspondence: Wei-hui Zeng: <zengweihui1965@163.com>

${ }^{*}$ These authors contributed equally to this work.

Received February 11, 2019 | Accepted February 5, 2020 
keratoses (7), Bowen's disease (8), and superficial basal cell carcinomas (9). In addition, PDT has been applied in the treatment with other types of human malignancies such as cervical cancer (10), breast cancer (11), prostate cancer (12), and glioma (13). With its range of indications continually expanding, PDT has also demonstrated potential as a treatment for cSCC (14).

PDT involves three essential components: visible-tonear-infrared (vis-NIR) light, a photosensitizer (PS), and sufficient amounts of oxygen (15). Diseased tissues can be highly selectively eradicated on activation of the non-toxic PS by irradiation. The second-generation PS 5-aminolevulinic acid (5-ALA) and its derivative methyl aminolevulinate have been approved by the Food and Drug Administration and are commonly used as topical PS (16). Although the use of 5-ALA-PDT for treating skin cancers is growing in popularity, a number of challenges must be overcome to improve the efficacy of PDT. Firstly, 5-ALA is hydrophilic and thus has limited ability to penetrate cell membranes, which restricts the effectiveness of topical 5ALA PDT. The low selectivity of 5-ALA for malignant cells and low levels of singlet oxygen generated also limit the clinical application of PDT (17). Furthermore, the instability of 5-ALA under physiological conditions reduce its photophysical properties and photodamage activity (18).

Gold nanoparticles (GNPs) possess a number of excellent features for use as drug delivery systems, including their tailorable size, stability, biocompatibility, and surface chemistry. Kim et al. (19) showed that tamoxifen conjugated to GNPs showed enhanced cell uptake by membranemediated diffusion and promoted the inhibitory effects of tamoxifen on breast cancer cells. Zhang et al. (20) developed a method for increasing paclitaxel solubility by covalent attachment to GNPs via DNA linkers, and the conjugates were more effective to inhibit viability of breast cancer cells and uterine sarcoma cells. Recently, Kwon et al. (21) demonstrated that Pu-18-N-butylimide-NMGA-GNP conjugated with PDT is effective against hepatocellular carcinoma progression.

In this study, we investigated whether 5-ALA-conjugated GNPs could enhance the anti-cancer efficiency of PDT in cutaneous squamous cell carcinoma as determined by in vitro functional assays and explored the underlying molecular mechanisms.

\section{Material and Methods}

\section{Synthesis of 5-ALA-GNPs}

GNPs were synthesized via the branched polyethylenimine (BPEI) method. To obtain positively charged GNPs, BPEl was used to reduce $\mathrm{HAuCl}_{4}$ into gold atoms and employed as a stabilizer. Briefly, $0.05 \mathrm{~g} \mathrm{BPEI}$ and $4 \mathrm{~mL} \mathrm{HAuCl}_{4}(25 \mathrm{mmol} / \mathrm{L})$ were mixed with ultrapure water (total volume, $50 \mathrm{~mL}$ ) at $80^{\circ} \mathrm{C}$, the solution was mixed until the color changed from yellow to dark red, and centrifuged at 25,000 $\mathrm{g}$ (CP $100 \mathrm{WX}, \mathrm{HITACHI}$, Japan) for $30 \mathrm{~min}$ at $4^{\circ} \mathrm{C}$ to pellet the GNPs. The supernatant was discarded and $10 \mathrm{~mL}$ ultrapure water was added to preserve the GNPs.

The 5-ALA solution was prepared by dissolving $0.0336 \mathrm{~g}$ 5-ALA in $2 \mathrm{~mL}$ ultrapure water to obtain a concentration of $50 \mathrm{mmol} / \mathrm{L}$ in the dark. The GNPs and 5-ALA were filtered through $0.22-\mu \mathrm{m}$ filters. The 5-ALA-GNPs were obtained by mixing 5-ALA and GNPs in a 1:2 ratio for $3 \mathrm{~min}$; HEPES $(20 \mathrm{mM})$ was used as a buffer to adjust the $\mathrm{pH}$ to 7.8 .

\section{Characterization of 5-ALA-GNPs}

The morphology of GNPs and 5-ALA-GNPs were investigated via high-resolution transmission electron microscopy (TEM; JEM-200CX, Hitachi, Japan). The diameter of the GNPs and the 5-ALA-GNPs were measured using a ZetaSizer Nano ZS90 instrument (Malvern Instruments, UK). The UV-Vis absorption spectra of GNPs and 5-ALA-GNPs were examined using an ultraviolet-visible spectrophotometer (DU-64, Jasco, Japan).

\section{Culture of epidermoid carcinoma A431 cells and HaCat cells}

A431 and HaCat cells were purchased from the Shanghai Cell Library of the Chinese Science Academy (China). A431 cells and HaCat cells were cultured in DMEM (Dulbecco's modified Eagle's medium, USA) containing $10 \%$ fetal bovine serum (FBS; Gibco, Thermo Fisher Scientific, USA), $100 \mathrm{U} / \mathrm{mL}$ penicillin, and $100 \mathrm{U} / \mathrm{mL}$ streptomycin at $37^{\circ} \mathrm{C}$ in a humidified atmosphere of $5 \% \mathrm{CO}_{2}$. The culture medium was refreshed every 2 days.

\section{In vitro PDT}

A431 cells or HaCat cells were seeded into 96-well plates in triplicate at $1 \times 10^{5}$ cells per well. Cells were incubated with phosphate-buffered saline (PBS), GNPs, 5 -ALA $(2,4$, and $8 \mathrm{mM})$ or 5-ALA-GNPs $(2,4$, and $8 \mathrm{mM})$ for $6 \mathrm{~h}$ in the dark, then irradiated at $621 \mathrm{~nm}$ using LEDs for $1.5 \mathrm{~h}$. A red LED light source (central wavelength $=621$ $\mathrm{nm}$; full width at half maximum $=15 \mathrm{~nm}$; luminous intensity 4000-5000 mcd; Xi'an Jiatong University, China) containing 96 LEDs with maximal emission to achieve a greater penetration depth and improve the efficacy of PDT was employed, and the energy fluency of the light sources was adjusted to $1 \mathrm{~mW} / \mathrm{cm}^{2}$ using a variable resistor in series.

\section{Morphology assessment and cell viability analysis (MTT assay and Alamar blue assay)}

At $24 \mathrm{~h}$ after irradiation, the morphology of the A431 cells and HaCat cells was observed via inverted microscopy (TE2000-U, Nikon, Japan). The MTT assay was employed to quantify cell viability. Briefly, $24 \mathrm{~h}$ after irradiation, the media in the 96-well plates was changed to $100 \mu \mathrm{L}$ drug-free DMEM medium and $20 \mu \mathrm{L}$ MTT $(5 \mathrm{mg} /$ $\mathrm{mL}$, Sigma, USA) and the cells were incubated in the dark for $4 \mathrm{~h}$. The media was then removed and $50 \mu \mathrm{L}$ of dimethyl sulfoxide (Sigma) was added to each well. Absorbance values were determined at $570 \mathrm{~nm}$ using a microplate 
reader (Wellscan MK3; Labsystems Dragon, Finland). For the Alamar blue assay, $24 \mathrm{~h}$ after irradiation, Alamar blue $(10 \% \mathrm{v} / \mathrm{v})$ was added for an additional $3 \mathrm{~h}$ before fluorescence was measured in triplicates for each sample with a fluorescence plate reader with excitation and emission at 560 and $590 \mathrm{~nm}$, respectively (Wellscan MK3).

\section{Apoptosis assay}

At $24 \mathrm{~h}$ after irradiation, the cells were harvested and incubated with $5 \mu \mathrm{L}$ of FITC-conjugated Annexin $\mathrm{V}$ and $5 \mu \mathrm{L}$ of propidium iodide for 15 min (Sigma) according to manufacturer's instructions at room temperature in the dark. The proportions of apoptotic cells were quantified using a FACS Calibur flow cytometer and Cellquest software (BD Biosciences, USA).

\section{Quantitative analysis of singlet oxygen generation}

Singlet oxygen sensor green reagent (SOSGR) was employed as a singlet oxygen-tracking agent to assess intracellular singlet oxygen generation. SOSGR emits green fluorescence at $525 \mathrm{~nm}$ in the presence of singlet oxygen. For the SOSGR assay, the cells were incubated with different reagents for $6 \mathrm{~h}$ in the dark. Samples were then centrifuged at $12,000 \mathrm{~g}$ for $5 \mathrm{~min}$ at $4^{\circ} \mathrm{C}$ followed by washing with PBS to remove remaining materials, and re-suspended in sodium dodecyl sulfate lysis buffer. The samples were then incubated for $20 \mathrm{~min}$ on ice and centrifuged at $12,000 \mathrm{~g}$ for $5 \mathrm{~min}$ at $4^{\circ} \mathrm{C}$. The suspension solutions of these centrifuged samples were collected and mixed with SOSGR reagent followed by light irradiation. SOSGR fluorescence was quantified at $24 \mathrm{~h}$ after irradiation using a fluorescence spectrophotometer (Hitachi F-4500, Japan) with a $525 \mathrm{~nm}$ excitation laser and near-infrared (NIR) detector (22).

\section{Quantitative real-time PCR analysis}

Total mRNA was isolated from cells by using TRIzol (Takara, China) and cDNA was synthesized using the SuperScript first-strand synthesis system (Invitrogen, USA). Relevant genes were amplified using the primers listed in Table 1 on an ABI 7500 detector (Applied Biosystems, USA) using the SYRB Green Master Mix kit (Takara). GAPDH was used as the internal reference for the relative expression of mRNA, and the relative expression levels of the genes were calculated by $2^{-\Delta \Delta C t}$ method.

\section{Western blot analysis}

Proteins from cells were extracted using the RIPA lysis buffer (Bio-Rad, USA) and the concentrations of the extracted proteins were measured using Bradford protein dye reagent (Bio-Rad) according to the manufacturer's protocol. Equal amounts of proteins were resolved by $10 \%$ SDS-PAGE and transferred to a PVDF membrane. After blocking with $5 \%$ non-fat milk at room temperature for $1 \mathrm{~h}$, the membrane was incubated with antibodies against STAT3, Bcl-2, Bax, $\beta$-catenin, c-myc, cyclin D1, and
Table 1. Primer sequences for qRT-PCR.

\begin{tabular}{|c|c|}
\hline Genes & Primer sequences \\
\hline STAT3 (forward) & 5'-GAGAAGCATTGTGAGTGAGC-3' \\
\hline STAT3 (reverse) & 5'-CGGTCCAGGCAGATGTTG-3' \\
\hline $\mathrm{Bcl}-2$ (forward) & 5'-TTTGAGTTCGGTGGGGTCATG-3' \\
\hline $\mathrm{Bcl}-2$ (reverse) & 5'-TCACTTGTGGCTCAGATAGGC-3' \\
\hline Bax (forward) & 5'-AACTGGTGCTCAAGGCCCTG-3' \\
\hline Bax (reverse) & 5'-GGGTGAGGAGGCTTGAGGAG-3' \\
\hline$\beta$-catenin (forward) & 5'-GCTTGGAATGAGACTGCTGA-3' \\
\hline$\beta$-catenin (reverse) & 5'-CTGGCCATATCCACCAGAGT-3' \\
\hline c-myc (forward) & 5'-AGCGACTCTGAGGAGGAACA-3' \\
\hline c-myc (reverse) & 5'-TCCAGCAGAAGGTGATCCA-3' \\
\hline cyclin D1 (forward) & 5'-TGCCACAGATGTGAAGTTCATT-3' \\
\hline cyclin D1 (reverse) & 5'-CAGTCCGGGTCACACTTGAT-3' \\
\hline GAPDH (forward) & 5'-CAAGGTCATCCATGACAACTTTG-3' \\
\hline GAPDH (reverse) & 5'-GTCCACCACCCTGTTGCTGTAG-3' \\
\hline
\end{tabular}

$\beta$-actin (Abcam, USA) at $4^{\circ} \mathrm{C}$ overnight. After that, the membrane was further incubated with respective horseradish peroxidase-conjugated secondary antibodies for $1 \mathrm{~h}$ at room temperature. The blots were visualized using an enhanced chemiluminescence kit (Thermo Fisher Scientific, USA) according to the manufacturer's protocol.

\section{Transwell invasion and migration assays}

Cell invasion and migration were evaluated using Transwell chambers (8- $\mu \mathrm{m}$ pore size; Millipore, USA). For the transwell invasion assay, the transwell inserts were pre-coated with Matrigel (Sigma), and for the transwell migration assay, the transwell inserts were without Matrigel. Briefly, cells were incubated with phosphate-buffered saline (PBS), GNPs, 5-ALA (8 mM), or 5-ALA-GNPs (8 mM) for $6 \mathrm{~h}$ in the dark, and $24 \mathrm{~h}$ after irradiation, the cells in $200 \mu \mathrm{L}$ serum-free medium were seeded onto the upper transwell chambers, while the lower transwell chambers were filled with $500 \mu \mathrm{L}$ medium supplemented with $10 \%$ FBS as a chemoattractant. After incubation for $24 \mathrm{~h}$ at $37^{\circ} \mathrm{C}$, the cells on the upper surface of the transwell chambers were removed by cotton wool, and the invaded and migrated cells on the lower surface were fixed with $70 \%$ ethanol followed by staining with $0.5 \%$ crystal violet. The number of invaded and migrated cells were counted under a light microscope by randomly selecting five fields.

\section{Statistical analysis}

Data were analyzed using GraphPad Prism 6.0 (GraphPad Software, USA). Data are reported as means \pm SD. Significant differences between treatment groups were analyzed using Student's $t$-test, one-way ANOVA (Bonferroni's post hoc test), or two-ANOVA (Bonferroni's post hoc test), as appropriate. $P$ values less than 0.05 were considered statistically significant. 


\section{Results}

\section{Characterization of 5-ALA-GNPs}

Transmission electron microscopy showed GNPs were uniform spheres with a smooth surface; agglomeration was not observed (Figure 1A, left panel). The 5-ALA-GNPs and GNPs had similar surface morphologies. However, 5-ALAGNPs had a larger diameter $(18.2 \pm 1.4 \mathrm{~nm})$ than GNPs $(16.1 \pm 1.1 \mathrm{~nm})$ (Figure 1A, right panel), indicating 5-ALA was effectively and stably combined with the GNPs.

The UV-Vis absorption spectra of GNPs and various concentrations of 5-ALA-GNPs were recorded (Figure 1B). The absorption peak for 5-ALA-GNPs at a 5-ALA concentration of $5 \mathrm{mmol} / \mathrm{L}$ was approximately $530 \mathrm{~nm}$. As the concentration of 5-ALA increased, the absorption peak tended to a red shift: the absorption peaks for 5-ALA-GNPs at 5 and $10 \mathrm{mmol} / \mathrm{L} 5-\mathrm{ALA}$ were similar, and the absorption peak for 5-ALA-GNPs at $20 \mathrm{mmol} / \mathrm{L}$ was about $550 \mathrm{~nm}$. This red shift indicated that the 5-ALA-GNP conjugates were unstable at high concentrations of 5-ALA. Thus, 5-ALA was prepared at concentrations of $2 \mathrm{mmol} / \mathrm{L}$, $4 \mathrm{mmol} / \mathrm{L}$, and $8 \mathrm{mmol} / \mathrm{L}$ for the subsequent in vitro tests.

\section{Morphology of HaCat and A431 cells}

Inverted light microscopy revealed that HaCat cells from the NC group expanded and gradually fused into each other, and the adherent cells were formed relatively homogeneous with a long spindle shape. GNPs treatment alone had no obvious effect on the morphology of HaCat cells compared to that from the NC group. PDT-treated (5-ALA or 5-ALA-GNPs) HaCat cells generally had a fusiform or polygonal shape without obvious pathological

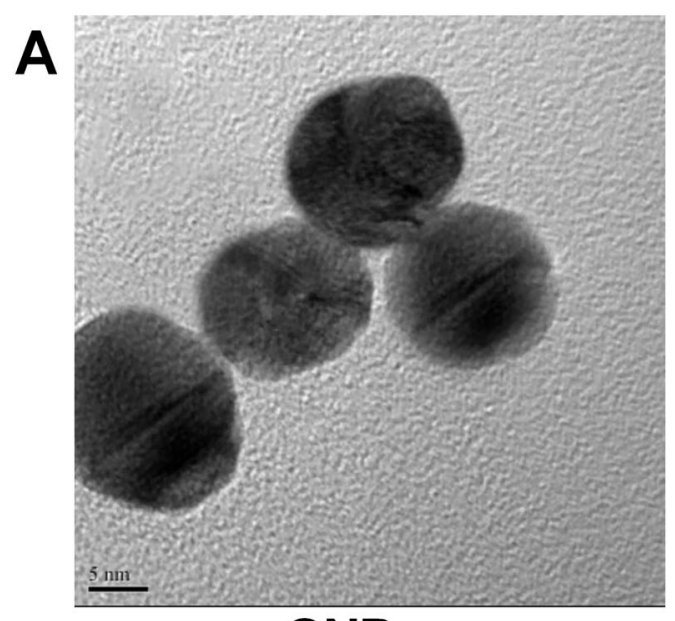

GNPs

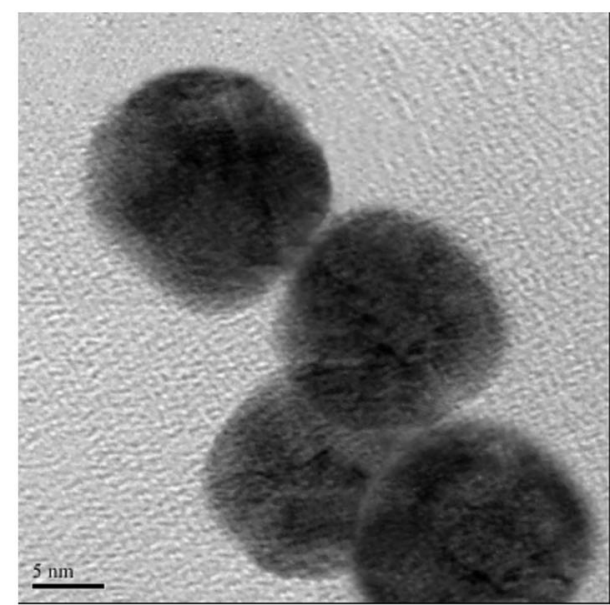

5-ALA-GNPs

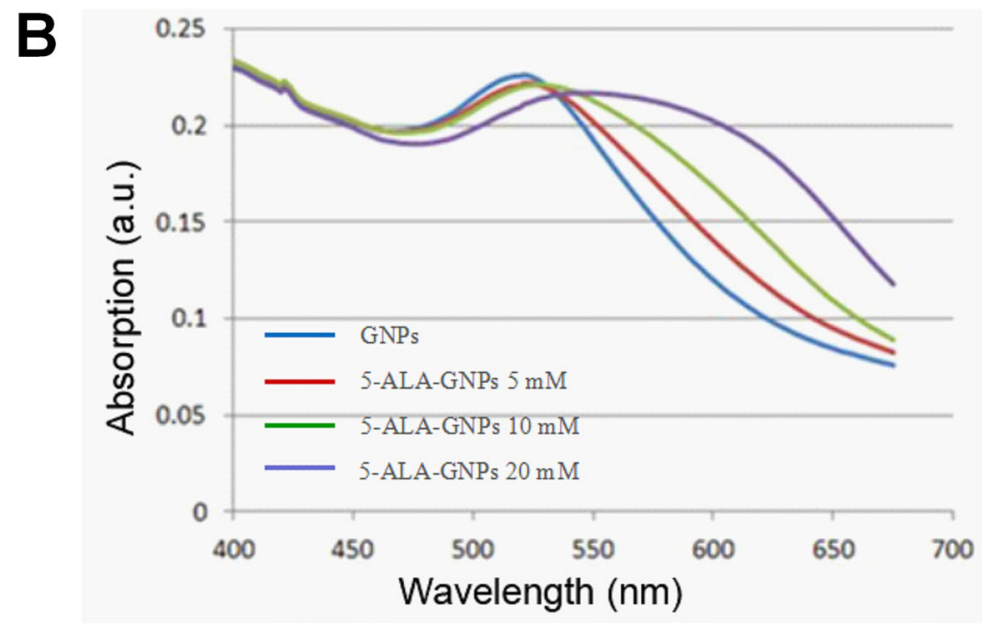

Figure 1. Transmission electron microscopy images and absorption spectra of gold nanoparticles (GNPs) and 5-aminolevulinic acid-(5ALA)-GNPs. A, Transmission electron microscopy images of GNPs and 5-ALA-GNPs. Scale bars, $5 \mathrm{~nm}$. B, Absorption spectra of GNPs and 5-ALA-GNPs. 
changes. However, cell debris and a reduction in cell volume were observed in the 5-ALA-GNPs (8 mM) group (Figure 2A).

The A431 cells from the NC group had a polygonal shape and were distributed evenly with clear boarders, and GNPs treatment had no significant effect on the morphology of A431 cells compared to those from the NC group. Cell shrinkage was observed in the 5-ALA (2, 4, and $8 \mathrm{mM}$ ) group. In the 5-ALA-GNPs group, nuclear pyknosis and cell shrinkage were observed, and the cells had an irregular polygonal shape. At high concentrations (4 and $8 \mathrm{mM}$ ) of 5-ALA-GNPs, the nuclei manifested the typical characteristics of apoptosis (Figure 2B).

Effects of in vitro PDT on cell viability, cell apoptosis, and singlet oxygen generation in HaCat and A431 cells

Treatment with different concentrations of 5-ALA for $6 \mathrm{~h}$ in the dark had no significant effect on the cell viability of HaCat and A431 cells (Supplementary Figure S1). In the PDT studies, HaCat and A431 cells were treated with PBS (NC), GNPs, 5-ALA (2, 4, and $8 \mathrm{mM}$ ), and 5-ALAGNPs $(2,4$, and $8 \mathrm{mM})$ for $6 \mathrm{~h}$ in the dark, and then irradiated at $628 \mathrm{~nm}$ for $1.5 \mathrm{~h}$. At $24 \mathrm{~h}$ after irradiation, cells were subjected to different in vitro functional assays. The MTT assay showed GNPs treatment had no significant effect on cell proliferative ability of both HaCat and A431 cells (data not shown). Flow cytometry showed no significant differences in cell apoptotic rates between NC and GNPs groups (Figure $3 \mathrm{~A}$ and $\mathrm{B}$ ). In addition, GNPs treatment did not affect the singlet oxygen generation of HaCat and A431 cells compared to the NC group (Figure $3 \mathrm{C}$ and D).

In the HaCat cells, PDT with 5-ALA suppressed cell viability in a concentration-dependent manner, with inhibitory rates being $11.23 \pm 1.22 \%$ ( $2 \mathrm{mM} \mathrm{5-ALA),} 17.23 \pm$ $1.44 \%$ (4 mM 5-ALA), and 30.65 $\pm 1.51 \%$ (8 mM 5-ALA),

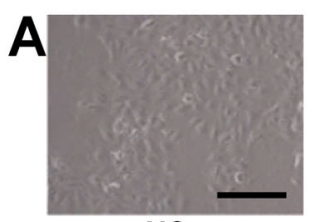

NC

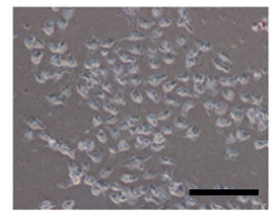

5-ALA-GNPs (2 mM)

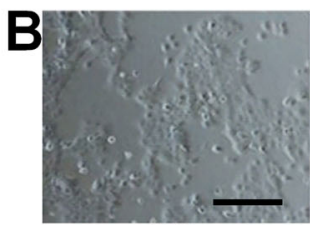

NC

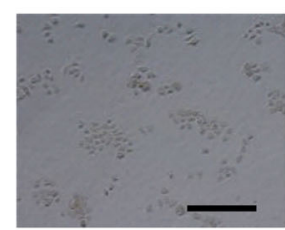

5-ALA (8 mM)

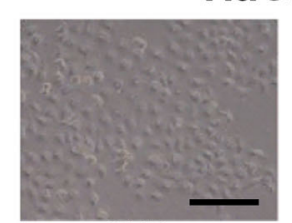

GNPs

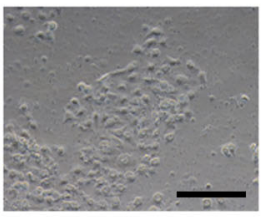

5-ALA-GNPs (4 mM)

A431

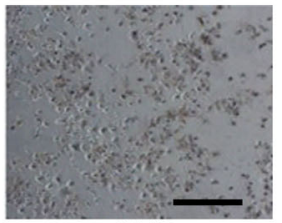

GNPs

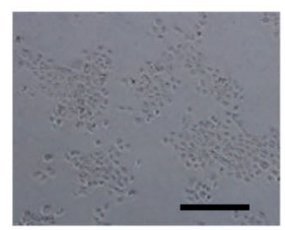

5-ALA-GNPs (2 mM)

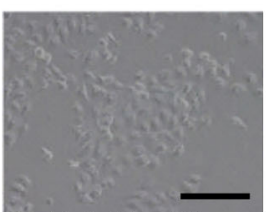

5-ALA (8 mM)
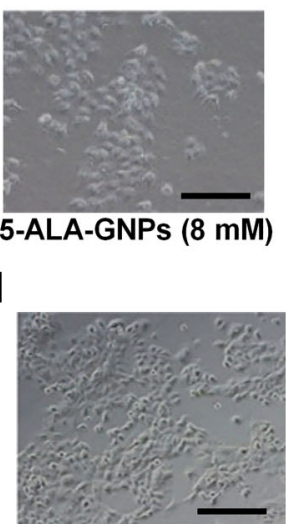

5-ALA (2 mM)

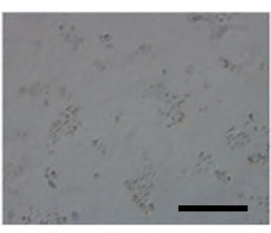

5-ALA-GNPs (4 mM)

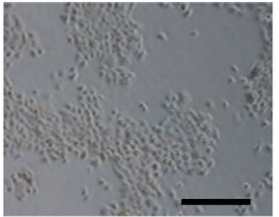

5-ALA (4 mM)

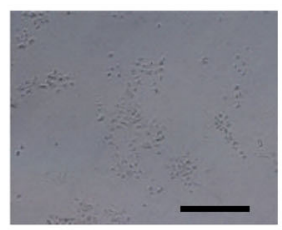

5-ALA-GNPs (8 mM)

Figure 2. Morphology of HaCat and A431 cells after in vitro photodynamic therapy (PDT) with 5-aminolevulinic acid (5-ALA) or 5-ALAgold nanoparticles (GNPs). A, Morphology of HaCat cells after being treated with phosphate-buffered saline (PBS) (negative control (NC)), GNPs, or in vitro PDT with 5-ALA ( $8 \mathrm{mM})$ or 5-ALA-GNPs $(2,4$, and $8 \mathrm{mM})$. Scale bars, $200 \mu \mathrm{m}$. B, Morphology of A431 cells after being treated with PBS (NC), GNPs, or in vitro PDT with 5-ALA $(2,4$, and $8 \mathrm{mM})$ or 5-ALA-GNPs $(2,4$, and $8 \mathrm{mM})$. Scale bars, $200 \mu \mathrm{m}$. 

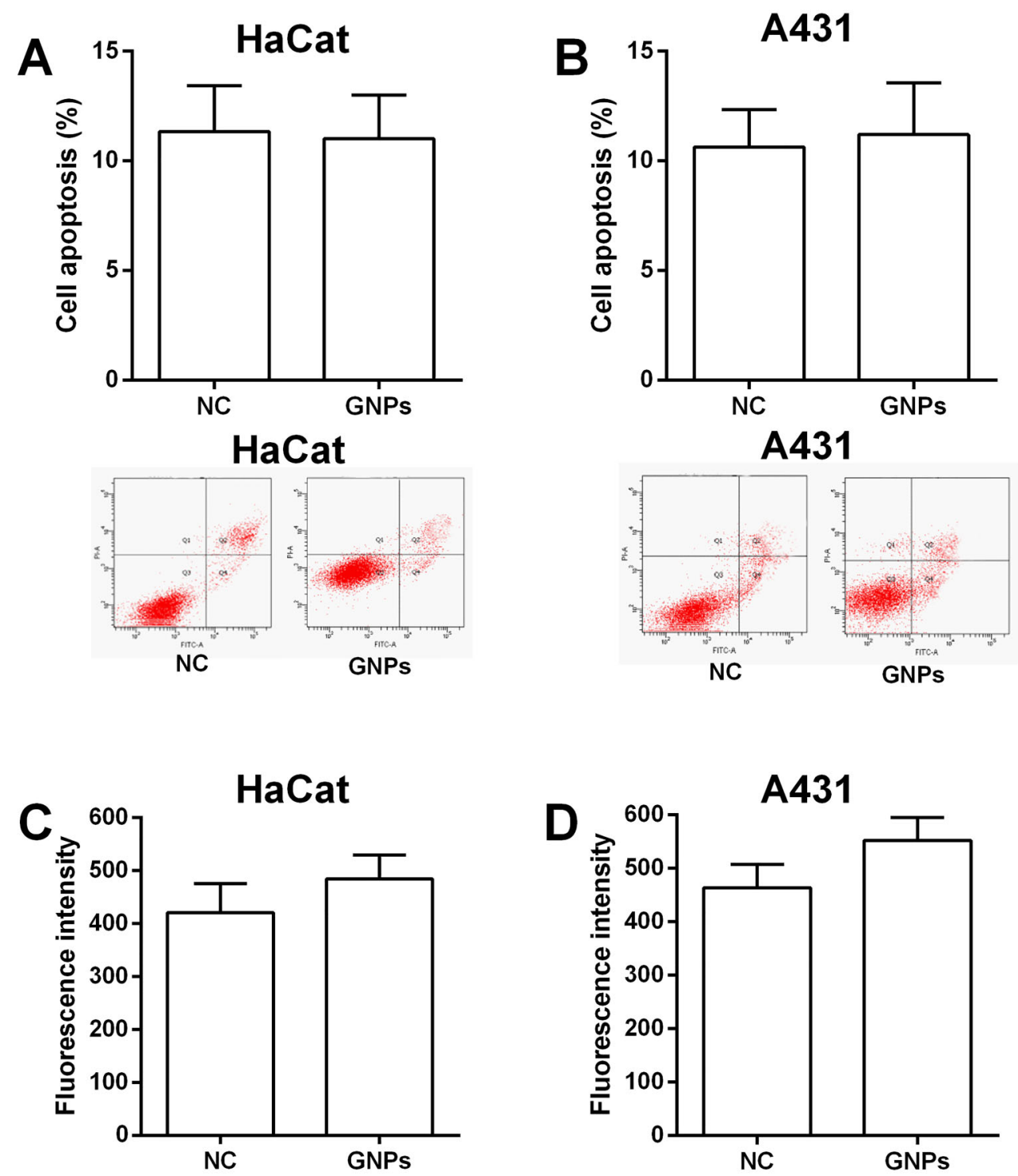

Figure 3. Effects of gold nanoparticles (GNPs) on cell apoptosis and singlet oxygen generation. Cell apoptosis of (A) HaCat and (B) A431 cells after being treated with phosphate-buffered saline (PBS (NC)) or GNPs was determined by flow cytometry (unpaired $t$-test). Singlet oxygen generation of (C) HaCat and (D) A431 cells after being treated with PBS (NC) or GNPs was determined by SOSGR assay (unpaired $t$-test). Data are reported as means \pm SD $(n=3)$.

while 5-ALA-GNPs groups also showed inhibitory rates of $12.93 \pm 1.97 \%$ (2 mM 5-ALA-GNPs), $19.62 \pm 2.01 \%$ (4 mM 5-ALA-GNPs), and 32.62 $\pm 2.33 \%$ (8 mM 5-ALAGNPs). However, no significant differences were found in the inhibitory rates between 5-ALA and 5-ALA-GNPS groups (Figure 4A). In the A431 cells, PDT with 5-ALA and 5-ALA-GNPs suppressed cell viability in a concentrationdependent manner, with inhibitory rates being much higher than that in HaCat cells (Figure 4B). Comparing the 5-ALA groups and the 5-ALA-GNPs groups with the same concentrations, the inhibitory rates in 5-ALA-GNPs groups were significantly higher than those in the 5-ALA groups (Figure 4B). The effects of different treatments on cell viability were further confirmed by the Alamar blue assay (Figure 4C and D). Cell apoptosis after PDT in $\mathrm{HaCat}$ and A431 cells was evaluated by flow cytometry. PDT with 5-ALA and 5-ALA-GNPs increased the cell apoptosis in HaCat and A431 cells in a concentrationdependent manner. Cell apoptotic rates of A431 cells after PDT with 5-ALA and 5-ALA GNPs were much higher than 

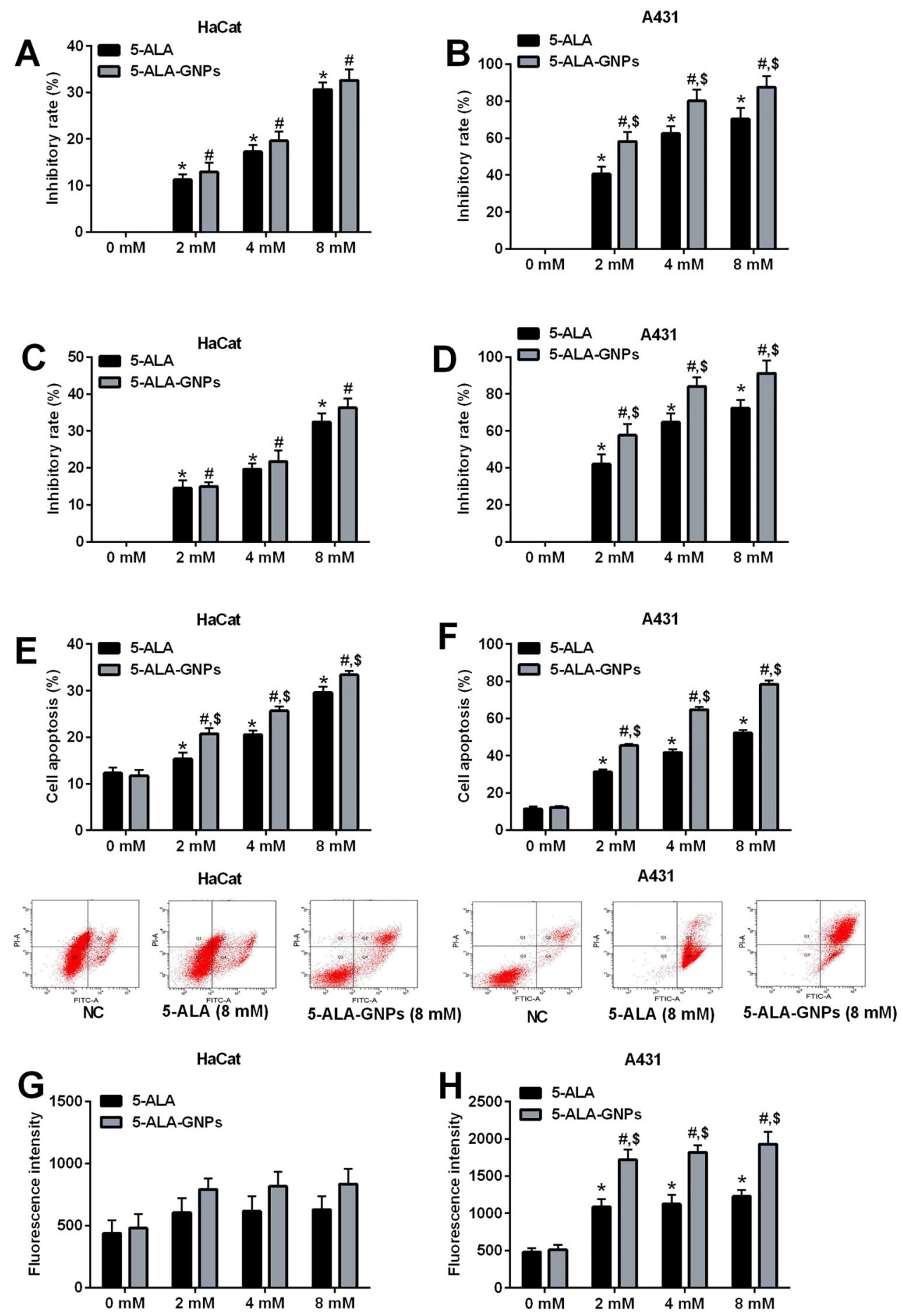

Figure 4. Effects of in vitro photodynamic therapy (PDT) on cell viability, cell apoptosis, and singlet oxygen generation in HaCat and A431 cells. Cell viability of (A) HaCat and (B) A431 cells after in vitro PDT with 5-aminolevulinic acid (5-ALA (2, 4, and 8 mM), 5-ALAgold nanoparticles (GNPs (2, 4, and $8 \mathrm{mM})$ ) or phosphate-buffered saline (PBS (NC)) were determined by MTT assay. Cell viability of (C) $\mathrm{HaCat}$ and (D) A431 cells after similar treatment were determined by Alamar blue assay. Cell apoptosis of (E) HaCat and (F) A431 cells after similar treatment were determined by flow cytometry. Singlet oxygen generation of $(\mathbf{G})$ HaCat and $(\mathbf{H})$ A431 cells after similar treatment were determined by SOSGR assay. Data are reported as means $\pm S D$. ${ }^{*} P<0.05$ compared to the 5 -ALA ( 0 mM) group; ${ }^{\#} \mathrm{P}<0.05$ compared to 5-ALA-GNPs $(0 \mathrm{mM})$ group; ${ }^{\$} \mathrm{P}<0.05,5-\mathrm{ALA}-\mathrm{GNPs}$ group vs 5-ALA group (two-way ANOVA, $\mathrm{n}=3$ ). 
those in HaCat cells. Additionally, 5-ALA-GNPs treatment significantly potentiated the effects of PDT on the cell apoptosis of HaCat and A431 cells compared to that with 5-ALA treatments (Figure 4E and F; Supplementary Figure S2).

PDT with 5-ALA and 5-ALA-GNPs had no significant effect on the singlet oxygen generation in HaCat cells (Figure 4G). On the other hand, PDT with 5-ALA increased the singlet oxygen generation in a concentration-dependent manner, with fluorescent intensity being $1086 \pm 107$ (2 mM 5-ALA), $1125 \pm 126$ (4 mM 5-ALA), and 1229 \pm 82 (8 mM 5-ALA), while PDT with 5-ALA-GNPs also significantly increased oxygen generation with fluorescent intensity being $1720 \pm 17$ (2 mM 5-ALA-GNPs), $1820 \pm 96$ (4 mM 5-ALA-GNPs), and $1930 \pm 162$ (8 mM 5-ALAGNPs) in a concentration-dependent manner (Figure $4 \mathrm{H}$ ). When comparing the 5-ALA and 5-ALA-GNPs groups with the same concentrations, the singlet oxygen generation levels in the 5-ALA-GNPs groups were significantly higher than those in the 5-ALA groups (Figure 4H).
Effects of in vitro PDT on expression of STAT3, Bcl-2, Bax, cell invasion, and migration in A431 cells

The effects of PDT on mRNA and protein expression of STAT3, Bcl-2, and Bax in A431 cells were determined by qRT-PCR and western blot assays, and PDT treatment with 5-ALA and 5-ALA-GNPs significantly suppressed the mRNA and protein expression of STAT3 and Bcl-2 and increased the mRNA and protein expression of Bax in A431 cells. In the 5-ALA-GNPs group, the mRNA and protein expression of STAT3 and Bcl-2 were significantly lower and the mRNA and protein expression of Bax were significantly higher than those in the 5-ALA group (Figure $5 \mathrm{~A}$ and $\mathrm{B}$ ).

The effects of PDT on cell invasion and migration of A431 cells were determined by transwell invasion and migration assays. PDT with 5-ALA and 5-ALA-GNPs both inhibited cell invasion and migration of A431 cells. In addition, the invasive and migratory potentials of A431 in the 5-ALA-GNPs group was much lower than those in 5-ALA group (Figure 5C and D).
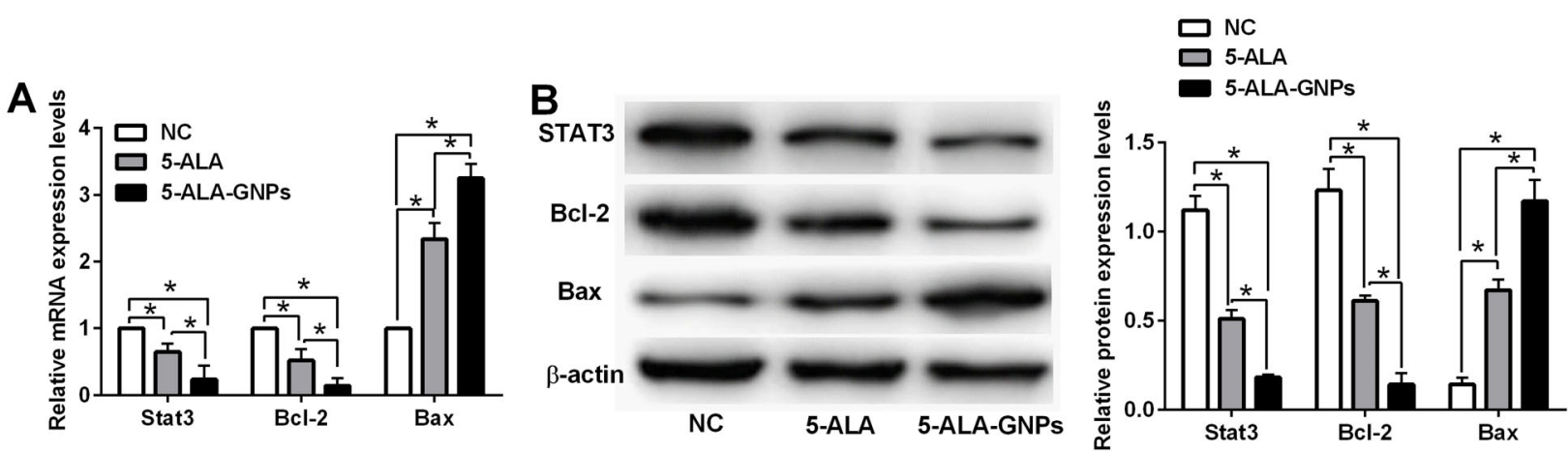

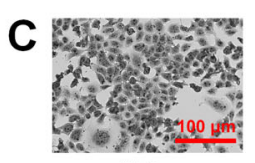

NC

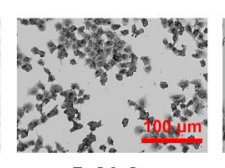

5-ALA
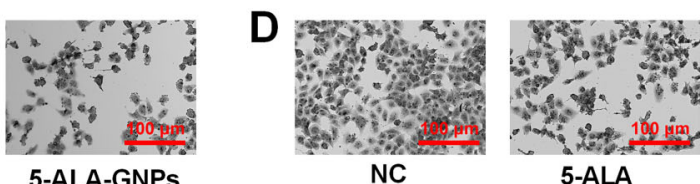

5-ALA
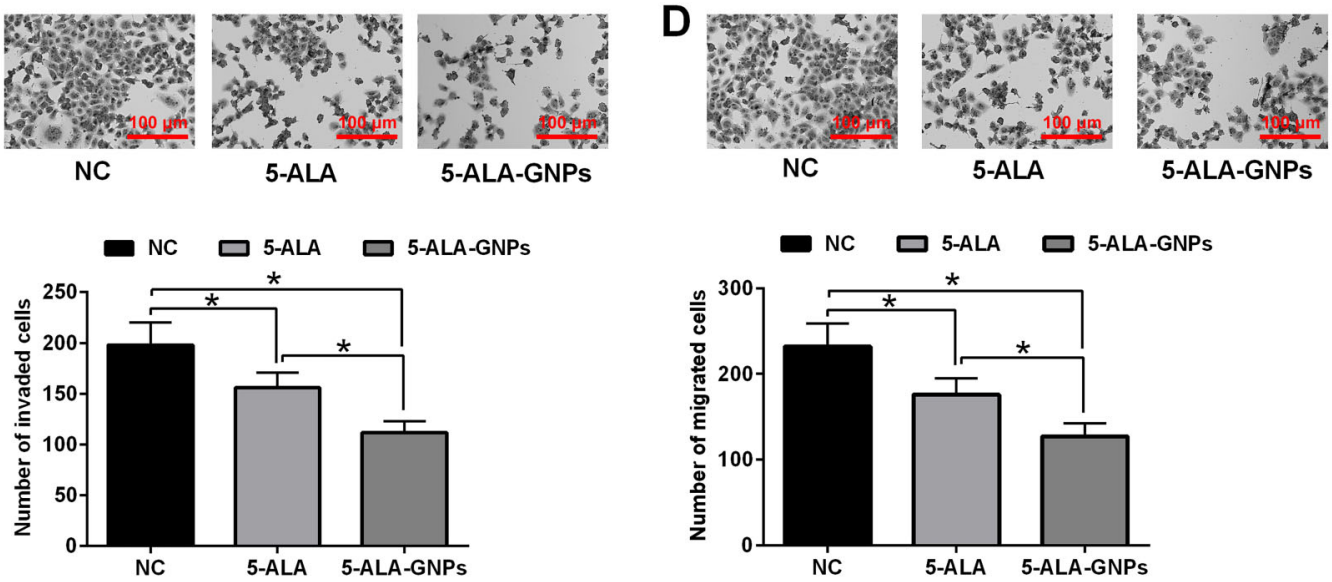

Figure 5. Effects of in vitro photodynamic therapy (PDT) on cell invasion and migration in $A 431$ cells. A, The mRNA expression and (B) protein expression of STAT3, Bcl-2, and Bax in A431 cells after in vitro PDT with 5-aminolevulinic acid (5-ALA) (4 mM), 5-ALA-gold nanoparticles (GNPs) (8 mM), or phosphate-buffered saline (PBS (NC)) was determined by qRT-PCR assay (one-way ANOVA). C and D, Cell invasion and migration of A431 cells after the same treatment were determined by transwell invasion and migration assays, respectively. Scale bars, $100 \mu \mathrm{m}$. Data are reported as means $\pm S D(n=3)$. Significant differences between groups are indicated as ${ }^{*} \mathrm{P}<0.05$ (one-way ANOVA). 

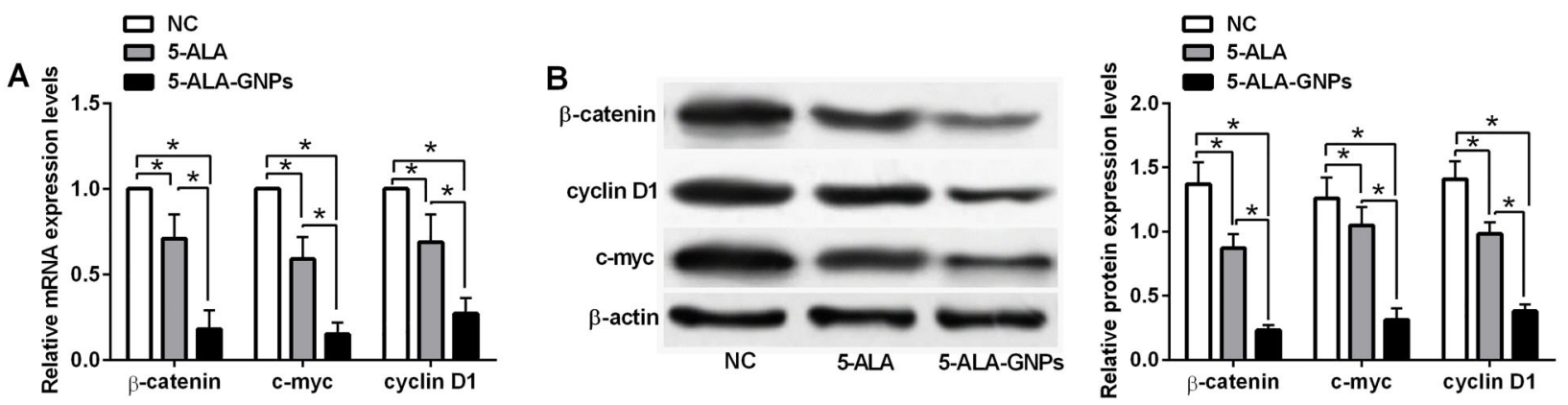

Figure 6. Effects of in vitro photodynamic therapy (PDT) on Wnt/ $\beta$-catenin signaling activities in A431 cells. A, mRNA expression and (B) protein expression of $\beta$-catenin, c-myc, and cyclin D1 in A431 cells after in vitro PDT with 5-aminolevulinic acid (5-ALA) (4 mM), 5ALA-gold nanoparticles (GNPs) (8 mM), or phosphate-buffered saline PBS (NC) was determined by qRT-PCR assay and western blot assay. Data are reported as means $\pm S D(n=3)$. Significant differences between groups are indicated as * $P<0.05$ (one-way ANOVA).

\section{Effects of in vitro PDT on Wnt/ $\beta$-catenin signaling activities in $\mathrm{A} 431$ cells}

The effects of PDT on the Wnt/ $\beta$-catenin signaling activities in A431 were evaluated by qRT-PCR and western blot assays. PDT with 5-ALA and 5-ALA-GNPs suppressed the mRNA and protein expression levels of $\beta$-catenin, c-myc, and cyclin D1 compared to the NC group in A431 cells. Furthermore, the suppressive effects on the expression of $W n t / \beta$-catenin signaling-related markers was potentiated in the 5-ALA-GNPs group compared to the 5-ALA group in A431 cells (Figure 6).

\section{Discussion}

PDT uses light to activate a non-toxic PS to generate reactive singlet oxygen, which mediates toxic effects in the target tumor cells. In clinical practice and in vitro, a number of parameters, including the concentration of the PS ALA, the ALA incubation time, and the dose of light are key factors that determine the cytotoxicity of ALAmediated PDT in tumor cells. Compared to other PSs, 5-ALA offers a number of advantages, such as low toxicity towards normal cells, its short clearance time, and the fact that it is rapidly converted to porphyrin (23). However, the hydrophilicity of 5-ALA and the low permeability of cell membranes to 5-ALA may limit the clinical efficiency of 5-ALA PDT (24). Furthermore, 5-ALA has poor stability under reductive biological conditions (25). Under alkaline conditions, 5-ALA loses hydrogen ions and becomes negatively charged. Thus, these polar characteristics of 5-ALA limit its ability to cross cell membranes. However, conjugation to biocompatible GNPs has been demonstrated to effectively deliver 5-ALA into target cells in vitro (23). The GNPs are reduced and become positively charged and are coated with BPEI, which makes 5-ALAGNPs relatively stable. In our study, no significant ALA was released from ALA-GNPs and the ALA-GNPs were kept stable at $4^{\circ} \mathrm{C}$ storage. Cheng et al. found that non-covalent drug/GNP conjugates could penetrate into tumors and lead to rapid drug release within hours (26). Zhang et al. (20) found that anti-cancer drugs conjugated to GNPs showed enhanced drug solubility and efficacy by increasing internalization of the conjugates. A recent study showed that 5-ALA conjugated to GNPs showed an enhanced uptake by the cancer cells compared to free 5-ALA (27). These results may imply that the increased tumor suppressive activities of the conjugates may be due to the increased uptake of 5-ALA by cancer cells.

Daniel et al. (28) demonstrated that delivery of a PS using a drug carrier significantly enhanced the inhibitory effects on tumor cell proliferation compared to the PS alone. In the present study, 5-ALA conjugated to GNPs improved the membrane permeability of 5-ALA, and 5-ALA-GNPs could stably accumulate in A431 cells and induce a dual photodynamic and photothermal effects, which could potentiate the anti-tumor effects of PDT in A431 cells.

PS selectivity is the most critical issue in PDT sensitizer design and efficiency. The higher the PS selectivity, the greater the accumulation of the PS in cancer cells, which reduces the side effects in normal cells (29). Therefore, $\mathrm{HaCat}$ cells were used to assess the effects of 5-ALA-GNPmediated PDT in normal cells. PDT using 5-ALA-GNPs led to fewer morphological changes, a significantly lower inhibitory rate, and significantly less apoptosis in HaCat cells than A431 cells. Moreover, the levels of singlet oxygen were significantly higher in A431 cells than HaCat cells, indicating that the 5-ALA-GNPs exerted selective effects in tumor cells.

PDT based on 5-ALA induces tumor cell death by both apoptotic and necrotic pathways (30). We showed that 5-ALA-GNPs effectively induced apoptosis in A431 cells in a concentration-dependent manner. Saczko et al. found that induction of apoptosis by PDT in a human melanoma cell line was dependent on photodynamic sensitivity, and that the DNA damages were determined by the PS concentration and duration of irradiation (31). We found that 5-ALA-GNPs induced significantly higher levels of 
apoptosis in A431 cells than HaCat cells, confirming the selective effects of 5-ALA-GNPs in tumor cells compared to normal cells. These findings imply that 5-ALA-GNPs could be used to selectively target tumor cells and have limited effects on normal cells.

PDT has been proposed to induce apoptosis in tumor cells by two mechanisms: 1) endogenous mechanisms, mediated by the loss of mitochondrial membrane integrity and increased mitochondrial membrane permeability; and 2) exogenous mechanisms, mediated by the endoplasmic reticulum stress-induced pathway (32). Zhang et al. (27) reported that the enhanced tumor cell-killing efficiency of 5-ALA-GNP conjugate-based PDT was mainly due to improved delivery of the PS to cells by the GNPs. In this study, 5-ALA-GNPs led to significantly higher singlet oxygen generation than 5-ALA. The conversion of 5-ALA into PpIX and generation of singlet oxygen mainly occur in the mitochondria, which is critical to improve efficiency and minimize unwanted cytotoxic activity of PDT (33). In this regard, improving mitochondria localization of 5-ALA is important. The enhanced membrane permeability of 5-ALA-GNPs plays a critical role in increasing the delivery of $P S$ into the mitochondria. 5-ALA-GNPs accumulate more efficiently in the mitochondria, at least in part, as positively charged and cationic GNPs are delocalized and have the potential to cross the inner mitochondrial membrane (34).

One possible mechanism of action of PDT is related to altered expression of Bcl-2 and Bax, which results in the inhibition of the JAK-STAT pathway (35). However, the specific mechanism by which 5-ALA-GNPs enhance PDT has not been fully elucidated. In this study, the flow cytometry results showed that cells treated with 5-ALA-GNPs after PDT had increased cell apoptotic rates compared to cells treated with 5-ALA after PDT, and the effect was enhanced with the increasing concentrations of 5-ALA. In addition, qRT-PCR and western blot were employed to explore the effects of 5-ALA-GNP-induced PDT in A431 cells. Compared to cells treated with 5-ALA, cells treated with 5-ALA-GNPs had significantly lower expression levels of STAT3 and Bcl-2 and higher expression levels of Bax, suggesting the induction of the apoptosis pathway

\section{References}

1. Gloster HM Jr, Neal K. Skin cancer in skin of color. $J A m$ Acad Dermatol 2006; 55: 741-760; quiz 761-764, doi: 10. 1016/j.jaad.2005.08.063.

2. Misak H, Zacharias N, Song Z, Hwang S, Man KP, Asmatulu $\mathrm{R}$, et al. Skin cancer treatment by albumin/5-Fu loaded magnetic nanocomposite spheres in a mouse model. $J$ Biotechnol 2013; 164: 130-136, doi: 10.1016/j.jbiotec.2013. 01.003.

3. Chakrabarty A, Geisse JK. Medical therapies for nonmelanoma skin cancer. Clin Dermatol 2004; 22: 183-188, doi: 10.1016/j.clindermatol.2003.12.005. and inhibition of the JAK-STAT pathway. In addition, 5-ALA-GNPs treatment enhanced the inhibitory effects of PDT on cell invasion and migration and Wnt/ $\beta$-catenin signaling activities in A431 cells compared to 5-ALA treatment. Jiang et al. (36), showed that PDT with hypocrellin $B$ inhibited cell migration in ovarian cancer. PDT also possesses inhibitor effects on cell invasion and migration of head and neck cancer cells in vitro (37). PDT with 5-ALA also induced phenotypic change and suppressed migration in human tongue squamous carcinoma cells (38). Wnt/ $\beta$-catenin signaling has been suggested for its important role in the cancer metastasis. Studies by Ma et al. (39) showed that nanoparticle delivery of Wnt-1 siRNA enhanced PDT by inhibiting the epithelialmesenchymal transmission for oral cancer. In addition, Dickkopf 3, a Wnt signaling inhibitor, intensified the antitumor effects of PDT on breast cancer cells (40). Collectively, these data may imply that 5-ALA-GNPs potentiate the inhibitory effects of PDT on A431 cell invasion and migration possibly via $\mathrm{Wnt} / \beta$-catenin signaling.

The present study demonstrated that 5-ALA conjugated to GNPs significantly enhanced the efficacy of 5ALA PDT in A431 cells. Furthermore, this study provided basic optimal parameters and a better understanding of the mechanism of action of 5-ALA-GNP-induced PDT in A431 cells. Further in vitro and clinical studies are required to determine the safety and efficacy of 5-ALA-GNPs as treatment for cutaneous squamous cell carcinoma. Indeed, novel PS functions in the tumor microenvironment need to be designed to improve the clinical efficacy of PDT and the outcomes of patients with cutaneous squamous cell carcinoma.

\section{Supplementary material}

Click here to view [pdf].

\section{Acknowledgment}

This study was supported by the National Natural Science Foundation of China (grant No. 81172590).

4. Caccialanza M, Piccinno R, Gaiani F, Contini D. Relevance of dermatologic radiotherapy in the therapeutic strategy of skin epithelial neoplasms: excellent results in the treatment of lesions localized on eyelids and skin overlying the cartilage of the nose. G Ital Dermatol Venereol 2013; 148: 83-88.

5. Veness $\mathrm{M}$, Richards $\mathrm{S}$. Role of modern radiotherapy in treating skin cancer. Australas J Dermatol 2003; 44: 159-66; quiz 167-178, doi: 10.1046/j.1440-0960.2003.06711.x.

6. Shi L, Wang X, Zhao F, Luan H, Tu Q, Huang Z, et al. In vitro evaluation of 5-aminolevulinic acid (ALA) loaded PLGA 
nanoparticles. Int J Nanomedicine 2013; 8: 2669-2676, doi: 10.2147/IJN.S45821.

7. Nguyen M, Sandhu SS, Sivamani RK. Clinical utility of daylight photodynamic therapy in the treatment of actinic keratosis - a review of the literature. Clin Cosmet Investig Dermatol 2019; 12: 427-435, doi: 10.2147/CCID.S167498.

8. Zaar O, Fougelberg J, Hermansson A, Gillstedt M, Wennberg-Larko AM, Paoli J. Effectiveness of photodynamic therapy in Bowen's disease: a retrospective observational study in 423 lesions. J Eur Acad Dermatol Venereol 2017; 31: 1289-1294, doi: 10.1111/jdv.14164.

9. de Albuquerque IO, Nunes J, Longo JP, Muehlmann LA, de Azevedo RB. Photodynamic therapy in superficial basal cell carcinoma treatment. Photodiagnosis Photodyn Ther 2019; 27: 428-432, doi: 10.1016/j.pdpdt.2019.07.017.

10. Chizenga EP, Chandran R, Abrahamse H. Photodynamic therapy of cervical cancer by eradication of cervical cancer cells and cervical cancer stem cells. Oncotarget 2019; 10: 4380-4396, doi: 10.18632/oncotarget.27029.

11. Monroe JD, Belekov E, Er AO, Smith ME. Anti-cancer photodynamic therapy properties of sulphur-doped graphene quantum dot and methylene blue preparations in MCF-7 breast cancer cell culture. Photochem Photobiol 2019; 95: 1473-1481, doi: 10.1111/php.13136.

12. Wang Q, Zhang X, Sun $Y$, Wang L, Ding L, Zhu WH, et al. Gold-caged copolymer nanoparticles as multimodal synergistic photodynamic/photothermal/chemotherapy platform against lethality androgen-resistant prostate cancer. Biomaterials 2019; 212: 73-86, doi: 10.1016/j.biomaterials.2019. 05.009.

13. Jia $Y$, Chen $L$, Chi $D$, Cong $D$, Zhou $P$, Jin J, et al. Photodynamic therapy combined with temozolomide inhibits C6 glioma migration and invasion and promotes mitochondrial-associated apoptosis by inhibiting sodium-hydrogen exchanger isoform 1. Photodiagnosis Photodyn Ther 2019; 26: 405-412, doi: 10.1016/j.pdpdt.2019.05.007.

14. Wang H, Li J, Lv T, Tu Q, Huang Z, Wang X. Therapeutic and immune effects of 5-aminolevulinic acid photodynamic therapy on UVB-induced squamous cell carcinomas in hairless mice. Exp Dermatol 2013; 22: 362-363, doi: 10. 1111/exd.12132.

15. Dolmans DE, Fukumura D, Jain RK. Photodynamic therapy for cancer. Nat Rev Cancer 2003; 3: 380-387, doi: 10.1038/ nrc1071.

16. Hopper C. Photodynamic therapy: a clinical reality in the treatment of cancer. Lancet Oncol 2000; 1: 212-219, doi: 10.1016/S1470-2045(00)00166-2.

17. Rodriguez L, Batlle A, Di Venosa G, MacRobert AJ, Battah $\mathrm{S}$, Daniel $\mathrm{H}$, et al. Study of the mechanisms of uptake of 5-aminolevulinic acid derivatives by PEPT1 and PEPT2 transporters as a tool to improve photodynamic therapy of tumours. Int J Biochem Cell Biol 2006; 38: 1530-1539, doi: 10.1016/j.biocel.2006.03.002.

18. Araújo LM, Thomazine JA, Lopez RF. Development of microemulsions to topically deliver 5-aminolevulinic acid in photodynamic therapy. Eur J Pharm Biopharm 2010; 75: 48-55, doi: 10.1016/j.ejpb.2010.01.008.

19. Kim CK, Ghosh P, Pagliuca C, Zhu ZJ, Menichetti S, Rotello VM. Entrapment of hydrophobic drugs in nanoparticle monolayers with efficient release into cancer cells. J Am Chem Soc 2009; 131: 1360-1361, doi: 10.1021/ja808137c.
20. Zhang $X Q, X u X$, Lam R, Giljohann D, Ho D, Mirkin CA. Strategy for increasing drug solubility and efficacy through covalent attachment to polyvalent DNA-nanoparticle conjugates. ACS Nano 2011; 5: 6962-6970, doi: 10.1021/ nn201446c.

21. Kwon JG, Song IS, Kim MS, Lee BH, Kim JH, Yoon I, et al. Pu-18-N-butylimide-NMGA-GNP conjugate is effective against hepatocellular carcinoma. Integr Med Res 2013; 2: 106-111, doi: 10.1016/j.imr.2013.05.001.

22. Xu H, Liu C, Mei J, Yao C, Wang S, Wang J, et al. Effects of light irradiation upon photodynamic therapy based on 5aminolevulinic acid-gold nanoparticle conjugates in K562 cells via singlet oxygen generation. Int $\mathrm{J}$ Nanomedicine 2012; 7: 5029-5038, doi: 10.2147/IJN.S33261.

23. Oo MK, Yang X, Du H, Wang H. 5-aminolevulinic acidconjugated gold nanoparticles for photodynamic therapy of cancer. Nanomedicine (Lond) 2008; 3: 777-786, doi: 10. 2217/17435889.3.6.777.

24. Baek S, Na K. A nano complex of hydrophilic phthalocyanine and polyethylenimine for improved cellular internalization efficiency and phototoxicity. Colloids Surf B Biointerfaces 2013; 101: 493-500, doi: 10.1016/j.colsurfb.2012.06.036.

25. Lee YD, Cho HJ, Choi MH, Park H, Bang J, Lee S, et al. Directed molecular assembly into a biocompatible photosensitizing nanocomplex for locoregional photodynamic therapy. J Control Release 2015; 209: 12-19, doi: 10.1016/ j.jconrel.2015.04.011.

26. Cheng Y, Meyers JD, Broome AM, Kenney ME, Basilion JP, Burda C. Deep penetration of a PDT drug into tumors by noncovalent drug-gold nanoparticle conjugates. J Am Chem Soc 2011; 133: 2583-2591, doi: 10.1021/ja108846h.

27. Zhang Z, Wang S, Xu H, Wang B, Yao C. Role of 5-aminolevulinic acid-conjugated gold nanoparticles for photodynamic therapy of cancer. J Biomed Opt 2015; 20: 51043, doi: 10.1117/1.JBO.20.5.051043.

28. Daniel MC, Astruc D. Gold nanoparticles: assembly, supramolecular chemistry, quantum-size-related properties, and applications toward biology, catalysis, and nanotechnology. Chem Rev 2004; 104: 293-346, doi: 10.1021/cr030698+.

29. Mehraban N, Freeman HS. Developments in PDT sensitizers for increased selectivity and singlet oxygen production. Materials (Basel) 2015; 8: 4421-4456, doi: 10.3390/ma807 4421.

30. Zhou F, Xing D, Chen WR. Regulation of HSP70 on activating macrophages using PDT-induced apoptotic cells. Int J Cancer 2009; 125: 1380-1389, doi: 10.1002/ijc.24520.

31. Saczko J, Kulbacka J, Chwilkowska A, Drag-Zalesiniska M, Wysocka T, Lugowski M, et al. The influence of photodynamic therapy on apoptosis in human melanoma cell line. Folia Histochem Cytobiol 2005; 43: 129-132.

32. Ji HT, Chien LT, Lin YH, Chien HF, Chen CT. 5-ALA mediated photodynamic therapy induces autophagic cell death via AMP-activated protein kinase. Mol Cancer 2010; 9: 91, doi: 10.1186/1476-4598-9-91

33. Shao W, Wang H, He S, Shi L, Peng K, Lin Y, et al. Photophysical properties and singlet oxygen generation of three sets of halogenated corroles. J Phys Chem B 2012; 116: 14228-14234, doi: 10.1021/jp306826p.

34. Pavani C, Uchoa AF, Oliveira CS, lamamoto Y, Baptista MS. Effect of zinc insertion and hydrophobicity on the membrane interactions and PDT activity of porphyrin photosensitizers. 
Photochem Photobiol Sci 2009; 8: 233-240, doi: 10.1039/ B810313E.

35. Qiao L, Mei Z, Yang Z, Li X, Cai H, Liu W. ALA-PDT inhibits proliferation and promotes apoptosis of SCC cells through STAT3 signal pathway. Photodiagnosis Photodyn Ther 2016; 14: 66-73, doi: 10.1016/j.pdpdt.2016.01.008.

36. Jiang $Y$, Leung AW, Wang $X$, Zhang $H, X u$ C. Effect of photodynamic therapy with hypocrellin $B$ on apoptosis, adhesion, and migration of cancer cells. Int J Radiat Biol 2014; 90: 575-579, doi: 10.3109/09553002.2014.906765.

37. Yang TH, Chen CT, Wang CP, Lou PJ. Photodynamic therapy suppresses the migration and invasion of head and neck cancer cells in vitro. Oral Oncol 2007; 43: 358-365, doi: 10.1016/j.oraloncology.2006.04.007.
38. Li PT, Ke ES, Chiang PC, Tsai T. ALA- or Ce6-PDT induced phenotypic change and suppressed migration in surviving cancer cells. J Dental Sci 2015; 10: 74-80, doi: 10.1016/j. jds.2013.10.005

39. Ma C, Shi L, Huang $Y$, Shen L, Peng H, Zhu X, et al. Nanoparticle delivery of Wnt-1 siRNA enhances photodynamic therapy by inhibiting epithelial-mesenchymal transition for oral cancer. Biomater Sci 2017; 5: 494-501, doi: 10.1039/C6BM00833J.

40. Mohammadpour H, Fekrazad R. Antitumor effect of combined Dkk-3 and 5-ALA mediated photodynamic therapy in breast cancer cell's colony. Photodiagnosis Photodyn Ther 2016; 14: 200-203, doi: 10.1016/j.pdpdt.2016. 04.001 . 